\title{
Dynamics of a Ramanujan-type Continued Fraction with Cyclic Coefficients
}

\author{
Jonathan M. Borwein * and D. Russell Luke ${ }^{\dagger}$
}

version 1.60 - June. 20, 2005

\begin{abstract}
We study several generalizations of the AGM continued fraction of Ramanujan inspired by a series of recent articles in which the validity of the AGM relation and the domain of convergence of the continued fraction were determined for certain complex parameters $[4,3,2]$. A study of the AGM continued fraction is equivalent to an analysis of the convergence of certain difference equations and the stability of dynamical systems. Using the matrix analytical tools developed in [2], we determine the convergence properties of deterministic difference equations and so divergence of their corresponding continued fractions.
\end{abstract}

AMS Subject Classification: 11J70, 40A15

KeYworDs: Continued fractions, Ramanujan AGM relation, difference equations, matrix analysis

\footnotetext{
${ }^{*}$ Faculty of Computer Science, Dalhousie University, Halifax, Nova Scotia B3H 1W5, Canada E-mail: jborwein@cs.dal.ca

${ }^{\dagger}$ Department of Mathematical Sciences, University of Delaware, Newark DE 19716-2553 E-mail: rluke@math.udel.edu
} 


\section{Introduction}

Let $a:=\left(a_{n}\right)_{n=1}^{\infty}$ be a sequence of nonzero complex-valued scalars bounded above and below, and let $b>1$. We denote the continued fraction $\mathcal{S}_{1}(a, b)$ by

$$
\mathcal{S}_{1}(a, b):=\frac{1^{b} a_{1}^{2}}{1+\frac{2^{b} a_{2}^{2}}{1+\frac{3^{b} a_{3}^{2}}{1+\ddots}} .}
$$

This is a generalization of a continued fraction studied by Ramanujan. Surprisingly, the original continued fracton, which can be written as $\mathcal{S}_{1}(a, 2)$ where $a=\left(a_{1}, a_{2}, a_{1}, a_{2}, \ldots\right)$ for $a_{1}$ and $a_{2}$ positive and real, satisfies an AGM relation, in addition to having applications to elliptic-function theory. Our interest in $\mathcal{S}_{1}(a, b)$ stems from investigations into the extent to which the properties of the original Ramanujan continued fraction can be generalized [4, 3, 2, 5]. Many special cases of the above continued fraction for particular choices of $a$ have been determined in $[3,2]$. In particular the cases (i) $a_{n}=$ const $\in \mathbb{C}$, (ii) $a_{n}=-a_{n+1} \in \mathbb{C}$, (iii) $\left|a_{2 n}\right|=1, a_{2 n+1}=i$, and (iv) $a_{2 n}=a_{2 m}, a_{2 n+1}=a_{2 m+1}$ with $\left|a_{n}\right|=\left|a_{m}\right| \forall m, n \in \mathbb{N}$.

In the present work we establish the following sufficient conditions for the divergence of $\mathcal{S}_{1}(a, b)$ with cyclic sequences of complex parameters $a=\left(a_{n}\right)_{n=1}^{\infty}$ of period $c<\infty$. This is a special case of the analysis of [5] in which random sequences of complex parameters were considered, but, as might be expected, the case of finite periodic sequences yields stronger results.

Theorem 1.1 (sufficient conditions for divergence) Let $b>1$, and let $a:=\left(a_{n}\right)$ be a sequence of nonzero complex parameters satisfying

$$
0 \neq \prod_{n=1}^{\infty}\left(1-\frac{1}{(2 n)^{b} a_{2 n}^{2}}\right)<\infty \quad \text { and } \quad 0 \neq \lim _{n \rightarrow \infty} \frac{a_{2}}{a_{2 n}^{2 n-1} a_{2 n-1}^{2 n-2}} \prod_{j=1}^{2 n-2} a_{j}^{2}<\infty
$$


Then the Ramanujan continued fraction $\mathcal{S}_{1}(a, b)$ defined by Eq.(1.1) diverges with the even/odd parts of $\mathcal{S}_{1}(a, b)$ converging to separate limits in the following cases:

(i) General deterministic parameters:

$$
\sup _{k}\left|\sum_{j \geq n}^{k} \frac{1}{a_{2}} \prod_{i=1}^{j} \frac{a_{2 i-1}^{2}}{a_{2 i}^{2}}\right|<\infty \quad \text { and } \quad \sup _{k}\left|\sum_{j \geq n}^{k} \frac{a_{2}}{a_{2 j}^{2}} \prod_{i=1}^{j} \frac{a_{2 i}^{2}}{a_{2 i-1}^{2}}\right|<\infty .
$$

(ii) Even periodic parameters: If $a_{n}=a_{n+c}$ for all $n$ and fixed $c$ even, and $|\gamma|=1$ with $\gamma \neq 1$ where

$$
\gamma:=\left(\prod_{n=1}^{c / 2} \frac{a_{2 n-1}^{2}}{a_{2 n}^{2}}\right)
$$

Part $(i)$ of Theorem 1.1 follows closely the stochastic analog [5, Theorem 1.1]. Parts $(i)$ and $(i i)$ are proven separately in section 4.3 .

For simplicity we work with the special case $\mathcal{S}_{1}(a, 2)$, which we will denote by either $\mathcal{S}_{1}(a)$ or simply $\mathcal{S}_{1}$. The more general case $\mathcal{S}_{1}(a, b)$ for $b>1$ is a straight forward generalization (see [5, Section 5]). Divergence of $\mathcal{S}_{1}$ is equivalent to convergence of the classical convergents $p_{n} / q_{n}$ to the fraction $\mathcal{S}_{1}$ where both $p_{n}$ and $q_{n}$ satisfy

$$
g_{n}=g_{n-1}+n^{2} \alpha_{n} g_{n-2} \quad \text { for } \quad \alpha_{n}:=a_{n}^{2},
$$

with $g$ standing in for either $p$ or $q$. We will use $\alpha_{n}$ and $a_{n}^{2}$ interchangeably throughout.

Anticipating the matrix analysis to follow, we reformulate Eq.(1.3) in terms of $2 \times 2$ matrices:

$$
\mathbf{q}_{n}=Q_{n} \mathbf{q}_{n-1} \quad \text { where } \quad Q_{n}:=\left[\begin{array}{cc}
1 & n^{2} \alpha_{n} \\
1 & 0
\end{array}\right] \quad \text { and } \quad \mathbf{q}_{n}:=\left(\begin{array}{c}
q_{n} \\
q_{n-1}
\end{array}\right)
$$

To analyze the case of cyclic parameters $a_{n}$ with periods of length $c$, we regroup the above recursion 
into blocks of length $c$

$$
\mathbf{q}_{c n}=\widehat{Q}_{n} \mathbf{q}_{c(n-1)} \quad \text { where } \quad \widehat{Q}_{n}:=\prod_{j=c(n-1)+1}^{c n} Q_{j} .
$$

We interpret the matrix product ascending from right to left.

We obtain sufficient conditions for the divergence of $\mathcal{S}_{1}$ through an analysis of rescaled difference equations derived from Eq.(1.3). Following [2] we consider the renormalized sequences $\left(t_{n}\right)$ and $\left(v_{n}\right)$ defined by

$$
t_{n}:=\frac{q_{n-1}}{n !} \quad \text { and } \quad v_{n}:=\frac{q_{n}}{\Gamma(n+3 / 2) a_{n}^{(n+1)}} .
$$

The sequence $\left(t_{n}\right)$ yields a well resolved analysis of cyclic parameters $a_{n}$ (odd or even), while the sequence $v_{n}$ is more convenient for a general, though less detailed, analysis.

To begin, we focus our attention in section 3 on cyclic parameters, that is $a_{n+c}=a_{n}$ for $c \geq 1$ and all $n$. In Theorem 3.1 we obtain upper bounds on the asymptotic behavior of $\left(t_{n}\right)$ under modest restrictions on the paramters $a_{n}$, in addition to lower bounds on the separation of the classical convergents of $\mathcal{S}_{1}$. The broader analysis of infinite sequences based on the sequence $\left(v_{n}\right)$, which we adapt from [5], is treated in section 4. This sequence is independent of the cycle length, though as we will see, there is a natural binary structure that allows one to obtain efficient formulations of the asymptotic behavior of the sequence and hence sufficient conditions for the divergence of $\mathcal{S}_{1}$. We prove Theorem 1.1 in section 4.3. Before presenting these main results, however, we report in section 4.2 partial results from an investigation of exponential sums and related integral representatioins along lines pursued in [2]. This analysis, while incomplete, provides some intriguing possibilities for further analysis of generalized Lerch sums. A summary of our most attractive results is given in Theorem 5. Before proceeding with the analysis, however, we motivate this study in section 2 with some numerical experiments of specific examples. 


\section{Numerical Motivation}

Following [5], we plot, for different cases of the parameters $a_{n}$ in the continued fraction $\mathcal{S}_{1}$, odd and even iterates of the recurrence

$$
t_{n}=\frac{1}{n} t_{n-1}+\frac{n-1}{n} \alpha_{n-1} t_{n-2}
$$

which follows from the rescaling Eq.(1.6). In [5, Eq.(1.10)] is was shown that for $\left|a_{n}\right|=\left|a_{m}\right|=b \neq 0$ for all $n, m \in \mathbb{N}$, the continued fraction $\mathcal{S}_{1}$ diverges if $\left(v_{n}\right)$ is bounded. There is an analogous, though less straight forward, condition for the divergence of $\mathcal{S}_{1}$ with respect to the sequence $\left(t_{n}\right)$. We derive this condition next.

By the standard identity [6, Eq.(1.2.10)], we have

$$
\begin{aligned}
\frac{p_{c n}}{q_{c n}}-\frac{p_{c n-1}}{q_{c n-1}} & =\frac{(-1)^{c n-1}(c n) !^{2}}{q_{c n} q_{c n-1}} \prod_{j=1}^{c n} a_{j}^{2} \\
& =\frac{(-1)^{c n-1}(c n) !^{2}}{q_{c n} q_{c n-1}}\left(\prod_{j=1}^{c} \alpha_{j}\right)^{n}
\end{aligned}
$$

thus

$$
\frac{p_{c n}}{q_{c n}}-\frac{p_{c n-1}}{q_{c n-1}}=\frac{(-1)^{c n-1}}{t_{c n+1} t_{c n}(c n+1)}\left(\prod_{j=1}^{c} \alpha_{j}\right)^{n}
$$

Hence, for $\left|a_{n}\right|=\left|a_{m}\right|=b \neq 0$ for all $n, m \in \mathbb{N}$, the continued fraction $\mathcal{S}_{1}$ diverges if

$$
\left|t_{n}\right| \leq O\left(\frac{b^{n}}{\sqrt{n}}\right)
$$

In our numerical experiments, we consider the case $\left|a_{n}\right|=1$ for all $n$.

In order to confirm the order of convergence of the iterates required by Eq.(2.4) (indicating the divergence of $\mathcal{S}_{1}$ ), we plot $\sqrt{n} t_{n}$. To begin, we reproduce in Fig. 1 the dynamics for periodic $\left(a_{n}\right)$ with cycle length 2 , and each $a_{1}$ and $a_{2}$ being roots of unity. This was also demonstrated in [2]. For 


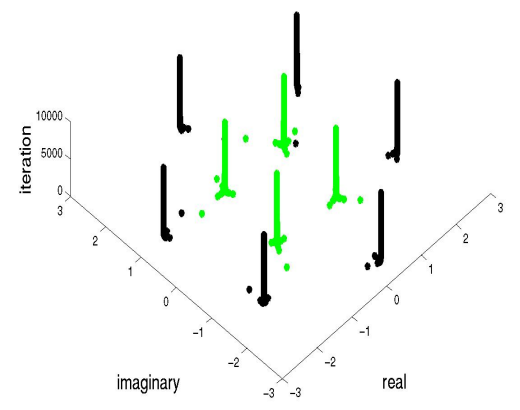

Figure 1: Dynamics for cycles of length $c=2$. Shown are the iterates $\widetilde{t}_{n}:=\sqrt{n} t_{n}$ for $t_{n}$ given by Eq.(2.1) with $\left(a_{1}, a_{2}\right)=(\exp (i \pi / 4), \exp (i \pi / 6))$. Odd iterates are light, even iterates are dark.

cycles of length $c=2 n(n \geq 2)$, the rates of convergence appear also to be $O(1 / \sqrt{n})$ with odd and even iterates easily distinguishable as shown in Fig. 2. These dynamics are explained principally in Section 3. As show in the analysis, the behavior of even cycles is remarkably regular.

When cycle lengths of $\left(a_{n}\right)$ are odd, however, the iterates of Eq.(2.1) display a much richer variety of behaviors. We show examples of cycles of length 3 with parameter values on the unit circle. The iterates still appear to obey a regular odd/even behavior, however in the first case Fig. 3 it appears that the iterates scaled by $\sqrt{n}$ are diverging. This indicates that the order of convergence of the unscaled iterates, if they converge at all, is something greater than $O(1 / \sqrt{n})$, which suggests, from Eq.(2.4), that it is possible that $\mathcal{S}_{1}$ converges for these parameters. On the other hand, for different parameter choices with cycles of length 3 shown in Fig. 4(a), it appears that the odd iterates scaled by $\sqrt{n}$ are converging, while the even iterates are diverging. In light of Eq.(2.4) it is unclear what this indicates about the continued fraction $\mathcal{S}_{1}$. We get a different picture if we look instead at the iterates $v_{n}$ of the corresponding recurrence

$$
v_{n}=\frac{2}{a_{n}(2 n+1)}\left(\frac{a_{n-1}}{a_{n}}\right)^{n} v_{n-1}+\frac{4 n^{2}}{(2 n-1)(2 n+1)}\left(\frac{a_{n-2}}{a_{n}}\right)^{(n-1)} v_{n-2} .
$$

As with Eq.(2.1) this recurrence follows directly from the rescaling Eq.(1.6). Fig. 4(b) shows the 


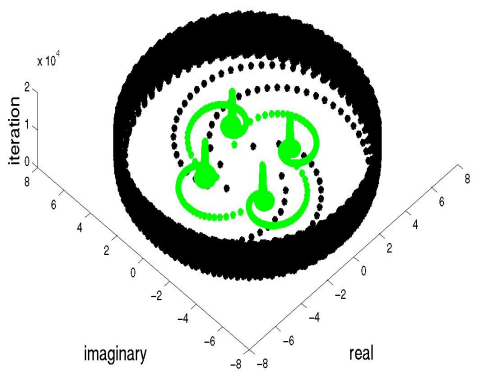

Figure 2: Dynamics for cycles of length $c=4$. Shown are the iterates $\widetilde{t}_{n}:=\sqrt{n} t_{n}$ for $t_{n}$ given by $E q$.(2.1) with cycle length $4, a_{1}=a_{3}=\exp (i \pi / 4), a_{2}=\exp (i \pi / 6), a_{4}=\exp (i(\pi / 6+1 / 2))$. Odd iterates are light, even iterates are dark.

unscaled iterates $v_{n}$. It appears from this simulation that the sequence $\left(v_{n}\right)$ is indeed bounded, though the iterates process around a circle of radius slightly larger than 1 in the complex plane. This indicates that the continued fraction $\mathcal{S}_{1}$ diverges for these parameter values. While it is not apparent from this example, the iterates $v_{n}$ display the same odd/even behavior as the rescaled iterates $t_{n}$. In our final example Fig. 5 we show the remarkable behavior of a length 3 cycle with well balanced parameters $a_{n}$. We explain exactly what we mean by "well balanced" in Section 4. In the first example, the scaled iterates of Eq.(2.1) appear to line up at specific locations in the complex plane. In the second example we see concentric orbits familiar from the even cycle examples.

While finite sequences of parameters display diverse behaviors that depend sensitively on whether the cycles are odd or even, random sequences are surprisingly robust. This remarkable behavior has been studied at length in [5].

Our object in the following analysis is to shed light on some of these dynamics. 


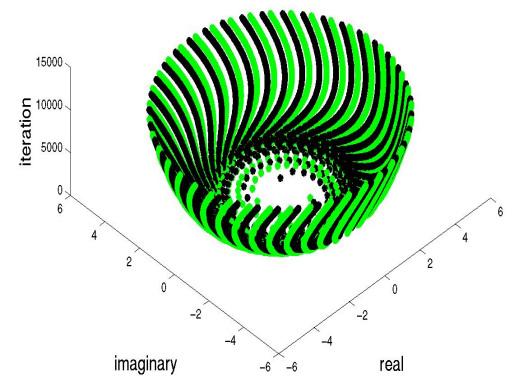

Figure 3: Dynamics for cycles of length 3. Shown are the iterates $\widetilde{t}_{n}:=\sqrt{n} t_{n}$ for $t_{n}$ given by Eq.(2.1) with $\left(a_{1}, a_{2}, a_{3}\right)=(\exp (i \pi / 4), \exp (i \pi / 4), \exp (i(\pi / 4+1 / \sqrt{2})))$. Odd iterates are light, even iterates are dark.

(a)

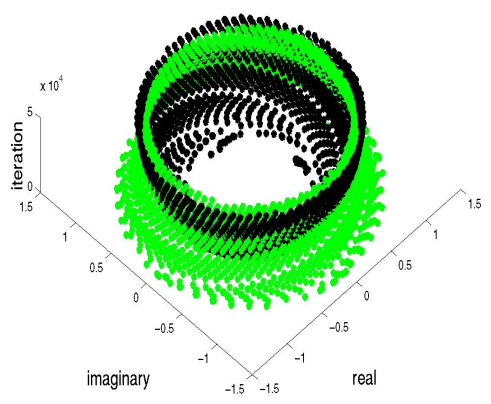

(b)

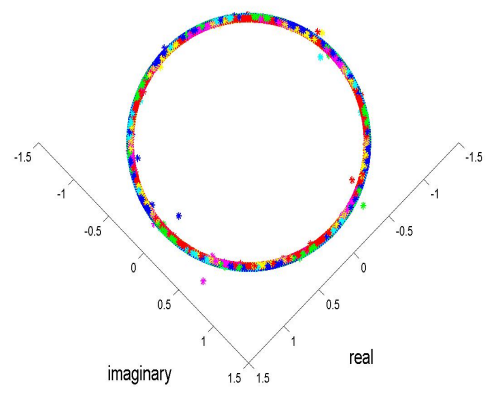

Figure 4: Dynamics for cycles of length 3. Shown are the iterates (a) $\widetilde{t}_{n}:=\sqrt{n} t_{n}$ for $t_{n}$ given by Eq.(2.1) and (b) $v_{n}$ given by Eq.(2.5). In both of these examples the parameter values are $\left(a_{1}, a_{2}, a_{3}\right)=(\exp (i \pi / 4),-\exp (i \pi / 4), \exp (i \pi / 4+1 / \sqrt{2}))$. Odd iterates are light, even iterates are dark. 


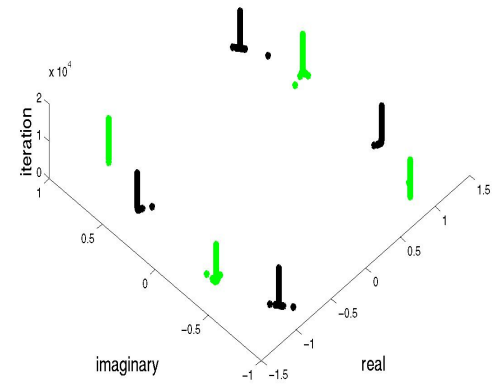

Figure 5: Dynamics for cycle of length $c=3$. Shown are the iterates $\widetilde{t}_{n}:=\sqrt{n} t_{n}$ for $t_{n}$ given by Eq.(2.1) with $\left(a_{1}, a_{2}, a_{3}\right)=(\exp (i \pi / 2), \exp (i \pi / 6), \exp (-i \pi / 6))$. Even iterates are light, odd iterates are dark.

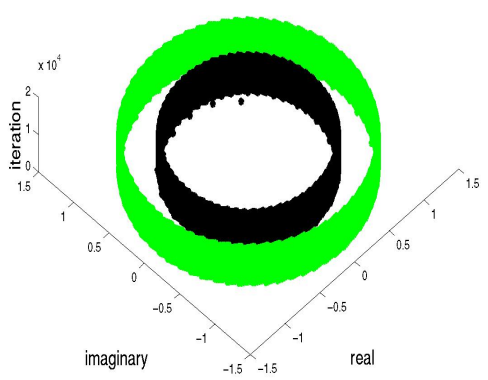

Figure 6: Dynamics for cycle of length $c=3$. Shown are the iterates $\widetilde{t}_{n}:=\sqrt{n} t_{n}$ for $t_{n}$ given by Eq.(2.1) with $\left(a_{1}, a_{2}, a_{3}\right)=(\exp (i(\pi / 3+0.05)), \exp (-i(\pi / 3+0.05)), \exp (0.05 i))$. Even iterates are light, odd iterates are dark. 


\section{Cyclic parameters: analysis of the renormalized sequence $\left(t_{n}\right)_{n=1}^{\infty}$}

As with $\mathbf{q}_{n}$ defined in Eq.(1.4), let

$$
\mathbf{t}_{n}:=\left(\begin{array}{c}
t_{n} \\
t_{n-1}
\end{array}\right)
$$

Then by Eq.(1.6)

$$
\mathbf{t}_{c n+1}=T_{n} \mathbf{t}_{(c(n-1)+1)} \quad \text { where } \quad T_{n}:=\left[N_{n}\right]^{-1} \widehat{Q}_{n} N_{n-1}
$$

for

$$
N_{n}:=\operatorname{Diag}((c n+1) !,(c n) !) .
$$

We look first at bounds on the sup-norm of the matrix $T_{n}$.

For parameter cycles $a_{n}=a_{n+c}$ of length $c$, induction on $c$ shows that

$$
\mathbf{t}_{c n}=T_{n} \mathbf{t}_{(c(n-1))}=\frac{2 n-2}{2 n-1}\left\{F_{n}+O\left(n^{-2}\right)\right\} \mathbf{t}_{(c(n-1))} .
$$

Here, for $c$ even,

$$
F_{n}:=\left[\begin{array}{cc}
\widetilde{\alpha}_{1} & \frac{1}{c n} \widetilde{\alpha}_{2} \\
\frac{1}{c n} \widetilde{\alpha}_{3} & \widetilde{\alpha}_{4}
\end{array}\right]
$$


with $^{1}$

$$
\begin{array}{ccc}
\widetilde{\alpha}_{1}:=\prod_{j=0}^{\frac{c}{2}-1} \alpha_{2 j}, & \widetilde{\alpha}_{2}:=\alpha_{1} \sum_{\substack{c-2 \geq j\left(\frac{c}{2}-1\right)-2 \geq \ldots \\
\cdots \geq j_{1} \geq 3}} \prod_{i=1}^{\frac{c}{2}-1} \alpha_{j_{i}}, \\
\widetilde{\alpha}_{3}:=\sum_{\substack{c-3 \geq j\left(\frac{c}{2}-1\right) \\
\cdots \geq j_{1} \geq 2}} \prod_{i=1}^{\frac{c}{2}-1} \alpha_{j_{i}} \quad \text { and } \quad \widetilde{\alpha}_{4}:=\prod_{j=0}^{\frac{c}{2}-1} \alpha_{2 j+1} .
\end{array}
$$

For $c$ odd, however,

$$
F_{n}:=\left[\begin{array}{cc}
\frac{1}{c n} \widehat{\alpha}_{1} & \left(1-\frac{1}{2 c n}\right) \widehat{\alpha}_{2} \\
\left(1+\frac{1}{2 c n}\right) \widehat{\alpha}_{3} & \frac{1}{c n} \widehat{\alpha}_{4}
\end{array}\right]
$$

with

$$
\begin{aligned}
\widehat{\alpha}_{1}:=\sum_{\substack{c-2 \geq j \\
\frac{c-1}{2}-2 \geq \ldots \\
\cdots \geq j_{1}-2 \geq 0}} \prod_{i=1}^{\frac{c-1}{2}} \alpha_{j_{i}}, \quad \widehat{\alpha}_{2}:=\prod_{j=0}^{\frac{c-1}{2}} \alpha_{2 j+1}, \\
\widehat{\alpha}_{3}:=\prod_{j=1}^{\frac{c-1}{2}} \alpha_{2 j}, \quad \text { and } \quad \widehat{\alpha}_{4}:=\alpha_{1} \sum_{\substack{c-3 \geq j \\
\frac{c-1}{2}-2 \geq \ldots \\
\cdots \geq j_{1}-2 \geq 1}} \prod_{i=1}^{\frac{c-1}{2}} \alpha_{j_{i}} .
\end{aligned}
$$

From Eq.(3.4) we obtain the bound

$$
\left|\mathbf{t}_{c N}\right| \leq\left(\prod_{n=2}^{N} \frac{2 n-2}{2 n-1}\left(\left|F_{n}\right|+O\left(n^{-2}\right)\right)\right)\left|\mathbf{t}_{c}\right| .
$$

\footnotetext{
${ }^{1}$ We define empty products to be equal to 1 .
} 
By the Wallis/Stirling formula [1] we know that

$$
\prod_{n=2}^{N} \frac{2 n-2}{2 n-1}=\sqrt{\frac{\pi}{4 N}}+O\left(N^{-3 / 2}\right)
$$

Moreover, the singular values of $F_{n}$ yield, to leading order,

$$
\left|F_{n}\right|= \begin{cases}\frac{1}{\sqrt{2}} \sqrt{\widetilde{b}_{1}+\left|\widetilde{b}_{2}\right|}+O\left(n^{-2}\right), & c \text { even and }\left|\widetilde{\alpha}_{1}\right| \neq\left|\widetilde{\alpha}_{4}\right| \\ \frac{1}{\sqrt{2}} \sqrt{\widehat{b}_{1}+\left|\widehat{b}_{2}\right|}\left(1+\frac{\widehat{b}_{2}}{2 c n\left|\widehat{b}_{2}\right|}\right)+O\left(n^{-2}\right), & c \text { odd and }\left|\widehat{\alpha}_{2}\right| \neq\left|\widehat{\alpha}_{3}\right|\end{cases}
$$

where

$$
\begin{array}{lll}
\widetilde{b}_{1}:=\left|\widetilde{\alpha}_{1}\right|^{2}+\left|\widetilde{\alpha}_{4}\right|^{2}, & \widetilde{b}_{2}:=\left|\widetilde{\alpha}_{1}\right|^{2}-\left|\widetilde{\alpha}_{4}\right|^{2}, \\
\widehat{b}_{1}:=\left|\widehat{\alpha}_{2}\right|^{2}+\left|\widehat{\alpha}_{3}\right|^{2} & \text { and } & \widehat{b}_{2}:=\left|\widehat{\alpha}_{2}\right|^{2}-\left|\widehat{\alpha}_{3}\right|^{2} .
\end{array}
$$

For $c$ even, then, if $\left|\widetilde{\alpha}_{1}\right| \neq\left|\widetilde{\alpha}_{4}\right|$, the behavior of the product of matrix norms is

$$
\prod_{n=2}^{N}\left(\left|F_{n}\right|+O\left(n^{-2}\right)\right)=O\left(\max \left(\left|\widetilde{\alpha}_{1}\right|,\left|\widetilde{\alpha}_{4}\right|\right)^{N}\right), \quad c \text { even. }
$$

For $c$ odd with $\widehat{b}_{2}<0$ we have

$$
\prod_{n=2}^{N}\left(\left|F_{n}\right|+O\left(n^{-2}\right)\right)=O\left(\frac{\left|\widehat{\alpha}_{3}\right|^{N}}{N^{1 / 2 c}}\right), \quad c \text { odd } .
$$

If, on the other hand, $\widehat{b}_{2}>0$, we see from Eq.(3.13) that the matrix product is unbounded as $N \rightarrow \infty$ and we lose any predictive power from this analysis. The behavior of the product of matrix norms apparently depends more intricately on the values of the sequence $\left(a_{n}\right)$ due to the $O\left(n^{-1}\right)$ term in Eq.(3.13). This also holds for the case $\left|\widetilde{\alpha}_{1}\right|=\left|\widetilde{\alpha}_{4}\right|$ or $\left|\widehat{\alpha}_{2}\right|=\left|\widehat{\alpha}_{3}\right|$ for $c$ even or odd 
respectively since, in this case,

$$
\left|F_{n}\right|= \begin{cases}\left|\widetilde{\alpha}_{1}\right|+\frac{1}{2 c n} \sqrt{\left|\widetilde{\alpha}_{1}\right|^{2}+\left|\widetilde{\alpha}_{2}\right|^{2}+\left|\widetilde{\alpha}_{3}\right|^{2}+2 \operatorname{Re}\left(\frac{\overline{\widetilde{\alpha}}_{1} \widetilde{\widetilde{\alpha}}_{2} \widetilde{\alpha}_{3}}{\widetilde{\alpha}_{4}}\right)}+O\left(n^{-2}\right), \quad\left|\widetilde{\alpha}_{1}\right|=\left|\widetilde{\alpha}_{4}\right| \\ \left|\widehat{\alpha}_{2}\right|+\frac{1}{2 c n} \sqrt{\left|\widehat{\alpha}_{1}\right|^{2}+\left|\widehat{\alpha}_{2}\right|^{2}+\left|\widehat{\alpha}_{4}\right|^{2}+2 \operatorname{Re}\left(\frac{\widehat{\alpha}_{1} \widehat{\alpha}_{2} \widehat{\alpha}_{4}}{\widehat{\alpha}_{3}}\right)}+O\left(n^{-2}\right), & \left|\widehat{\alpha}_{2}\right|=\left|\widehat{\alpha}_{3}\right| .\end{cases}
$$

A straight forward calculation shows that the $O\left(n^{-1}\right)$ term in Eq.(3.16) disappears only when $F_{n}$ is the trivial zero matrix, hence there are no nontrivial sequences $\left(a_{n}\right)$ for which the product of matrices converges.

We summarize this discussion with the following theorem which generalizes [2, Theorem 5.1].

Theorem 3.1 (convergence/divergence rates for cyclic parameters) Let the coefficients of the continued fraction $\mathcal{S}_{1}(a)$ be given by $a=\left(a_{1}, a_{2}, \ldots, a_{c}\right) \in \mathbb{C}^{c}$. For c even with $\left|\widetilde{\alpha}_{1}\right| \neq\left|\widetilde{\alpha}_{4}\right|$, and $\widetilde{\alpha}_{j}$ defined by Eq.(3.6)-Eq.(3.7), any solution of the recurrence Eq.(2.3) has the asymptotic behavior

$$
\left|t_{c n}\right| \leq O\left(\frac{\max \left(\left|\widetilde{\alpha}_{1}\right|,\left|\widetilde{\alpha}_{4}\right|\right)^{n}}{\sqrt{n}}\right)
$$

and the convergents to $\mathcal{S}_{1}(a)$ satisfy, for $\gamma>0$ constant,

$$
\left|\frac{p_{2 c n}}{q_{2 c n}}-\frac{p_{2 c n-1}}{q_{2 c n-1}}\right| \geq \gamma \min \left(\left|\frac{\widetilde{\alpha}_{4}}{\widetilde{\alpha}_{1}}\right|,\left|\frac{\widetilde{\alpha}_{1}}{\widetilde{\alpha}_{4}}\right|\right)^{n}
$$

For $c$ odd and $\left|\widehat{\alpha}_{2}\right|<\left|\widehat{\alpha}_{3}\right|$ where $\widehat{\alpha}_{j}$ are defined by Eq.(3.9)-Eq.(3.10), any solution of recurrence Eq.(2.3) has the asymptotic behavior

$$
\left|t_{c n}\right| \leq O\left(\frac{\left|\widehat{\alpha}_{2}\right|^{n}}{n^{(c+1) /(2 c)}}\right)
$$

and the convergents to $\mathcal{S}_{1}(a)$ satisfy, for $\gamma>0$ constant,

$$
\left|\frac{p_{2 c n}}{q_{2 c n}}-\frac{p_{2 c n-1}}{q_{2 c n-1}}\right| \geq \gamma\left(\frac{\left|\widehat{\alpha}_{3}\right|}{\left|\widehat{\alpha}_{2}\right|}\right)^{n} n^{1 / c}
$$




\section{General deterministic parameters: analysis of the renormalized sequence $\left(v_{n}\right)_{n=1}^{\infty}$}

The analysis of the previous section provides upper bounds on the rate of convergence of the sequence $\left(t_{n}\right)$ for $\left|\widetilde{\alpha}_{1}\right| \neq\left|\widetilde{\alpha}_{4}\right|$ in the case of cycles of even length, and for odd-length cycles when $\left|\widehat{\alpha}_{2}\right|>\left|\widehat{\alpha}_{3}\right|$. The case $\left|\widetilde{\alpha}_{1}\right|=\left|\widetilde{\alpha}_{4}\right|$ for periodic $\left(a_{n}\right)$, and, more generally, the case of infinite sequences $\left(a_{n}\right)$ requires different analytical tools which we study in this section.

\subsection{Matrix Products}

Define

$$
\mathbf{v}_{n}:=\left(\begin{array}{c}
v_{n} \\
v_{n-1}
\end{array}\right) .
$$

Then by Eq.(1.6)

$$
\mathbf{v}_{n}=Y_{n} \mathbf{v}_{n-1} \quad \text { where } \quad Y_{n}:=G_{n}^{-1} Q_{n} G_{n-1}
$$

for

$$
G_{n}:=\operatorname{Diag}\left(\Gamma\left(n+\frac{3}{2}\right) a_{n}^{(n+1)}, \Gamma\left(n+\frac{1}{2}\right) a_{n-1}^{n}\right) .
$$

We pursue here a matrix analysis of $\mathcal{S}_{1}$ based on the renormalized sequence $\left(\mathbf{v}_{n}\right)$.

Though the basic framework of our analysis makes no use of the notion of a cycle, the sequence $\left(\mathbf{v}_{n}\right)$ still exhibits an odd/even behavior which we can isolate by looking at every second iterate of $\left(\mathbf{v}_{n}\right)$ or, equivalently, by studying the matrix $\widehat{Y}_{n}$ defined by

$$
\widehat{Y}_{n}:=Y_{2 n} Y_{2 n-1}
$$

where $Y_{n}$ is given in Eq.(4.2). The next theorem establishes the relation between the convergence of

the infinite product of matrices $\widehat{Y}_{n}$ and the divergence of $\mathcal{S}_{1}$. This is a restatement of [5, Theorem 
4.1], to which we refer the interested reader for proof and further discussion.

Theorem 4.1 (odd and even convergents of continued fractions) If the matrix

$$
\mathcal{Y}_{n}:=\prod_{j=1}^{n} \widehat{Y}_{n}
$$

converges to a finite matrix $\mathcal{Y}^{\infty}$ with nonzero eigenvalues, then for the standard initial conditions

$$
\left(u_{-1}, u_{0}, v_{-1}, v_{0}\right)=\left(\frac{1}{\sqrt{\pi}}, 0,0, \frac{2}{a_{0} \sqrt{\pi}}\right)
$$

the even and odd parts of $\mathcal{S}_{1}(a)$ are given by

$$
\mathcal{S}_{1}^{(\text {even })}(a)=\frac{a_{0} y_{1,2}^{\infty}}{2 y_{1,1}^{\infty}}, \quad \text { and } \quad \mathcal{S}_{1}^{(\text {odd })}(a)=\frac{a_{0} y_{2,2}^{\infty}}{2 y_{2,1}^{\infty}}
$$

where $y_{i, j}^{\infty}$ is the $i, j$ th element of $\mathcal{Y}^{\infty}$. These limits are not equal, thus $\mathcal{S}_{1}$ diverges. The separation of odd and even limits is given explicitly by

$$
\mathcal{S}_{1}^{(\text {even })}(a)-\mathcal{S}_{1}^{(\text {odd })}(a)=-\frac{a_{0}^{2}}{2 a_{2} y_{1,1}^{\infty} y_{2,1}^{\infty}} \operatorname{det}\left(\mathcal{Y}^{\infty}\right)
$$

What remains, then, is to determine the conditions under which $\mathcal{Y}_{n}$ converges as $n \rightarrow \infty$. Following [5] we extract the leading-order behavior. Implicit in the discussion to this point is that the coefficients $a_{n}$ are nonzero and bounded. Thus, for each $n$, the components $\widehat{y}_{i j}^{(n)}$ of $\widehat{Y}_{n}$ are bounded, so we can expand $\widehat{Y}_{n}$ in powers of $n^{-1}$ to get

$$
\mathcal{Y}_{n}=\mathcal{U}_{n}+O\left(n^{-2}\right)
$$

where

$$
\mathcal{U}_{n}=\left(\prod_{j=2}^{n} K_{j}\right) \prod_{j=2}^{n}\left(I+\frac{1}{2 j} \widehat{W}_{j}\right)
$$


for

$$
K_{n}=\left[\begin{array}{cc}
\left(\frac{\alpha_{2 n-2}}{\alpha_{2 n}}\right)^{n-1 / 2} & 0 \\
0 & \left(\frac{\alpha_{2 n-3}}{\alpha_{2 n-1}}\right)^{n-1}
\end{array}\right]
$$

and

$$
\widehat{W}_{n}=\frac{1}{a_{2 n}}\left[\begin{array}{cc}
0 & \omega_{n} \\
\omega_{n}^{-1} & 0
\end{array}\right], \quad \text { with } \quad \omega_{n}=\frac{a_{2 n}}{a_{2}} \prod_{j=1}^{n} \frac{\alpha_{2 j-1}}{\alpha_{2 j}} .
$$

Remark 4.2 At this point one can easily see how the parity of the cycle lengths (odd or even) might have a profound impact on the dynamics of the recursion for the classical convergents of $\mathcal{S}_{1}$. For cycle length $c$ even we have

$$
\omega_{n}=\frac{a_{2 n \bmod c}}{a_{2}}\left(\prod_{j=1}^{n \bmod c / 2} \frac{\alpha_{2 j-1}}{\alpha_{2 j}}\right)\left(\prod_{j=1}^{c / 2} \frac{\alpha_{2 j-1}}{\alpha_{2 j}}\right)^{\lfloor 2 n / c\rfloor} \quad(c \text { even })
$$

For odd-length cycles, on the other hand, the power disappears through cancellation:

$$
\omega_{n}=\frac{a_{2 n \bmod c}}{a_{2}} \prod_{j=1}^{n \bmod c} \frac{\alpha_{2 j-1}}{\alpha_{2 j}} \quad(c \text { odd })
$$

We will return to this in the next sections.

To ease the computations, we focus our attention on the rotated product

$$
\widehat{U}_{n}:=\left(\prod_{j=2}^{n} K_{j}\right)^{-1} \mathcal{U}_{n}=\prod_{j=2}^{n}\left(I+\frac{1}{2 j} \widehat{W}_{j}\right)
$$

The justification for this follows next.

Theorem 4.3 (invertible matrix products) Let $\left(A_{n}\right)$ and $\left(C_{n}\right)$ be sequences of $m \times m$ complex matrices. Suppose that (i) $\left(\prod_{j=1}^{n} C_{j} \prod_{j=1}^{n} A_{j}\right)$ converges to the invertible matrix $L_{1}$ as $n \rightarrow \infty$, and (ii) $\prod_{j=1}^{n} C_{n}$ converges to an invertible matrix $L_{2}$. Then, as $n \rightarrow \infty$, the matrix product $\prod_{j=1}^{n} A_{n}$ converges to $L_{2}^{-1} L_{1}$. Moreover, if $\left(B_{n}\right)$ is a sequence of $m \times m$ complex matrices satisfying (iii) 
$\sum_{j=1}^{\infty}\left|B_{j}\right|<\infty$, then $\prod_{j=1}^{n}\left(A_{j}+B_{j}\right)$ converges to a finite complex matrix.

Proof. By assumption (i), given any $\epsilon$ there is an $N_{1}$ such that $n \geq N_{1}$ implies that

$$
\left|\prod_{j=1}^{n} C_{j} \prod_{j=1}^{n} A_{j}-L_{1}\right|<\frac{\epsilon}{2 M}
$$

where $M \geq \max _{k \geq N_{1}}\left|\left(\prod_{j=1}^{k} C_{j}\right)^{-1}\right|$ (which exists by (ii)). The Cauchy-Schwarz inequality then yields

$$
\left|\prod_{j=1}^{n} A_{j}-\left(\prod_{j=1}^{n} C_{j}\right)^{-1} L_{1}\right|<\frac{\epsilon}{2}
$$

On the other hand, by (ii) and [2, Lemma 6.3b], $\left(C_{j}\right)$ is tail Cauchy, that is, $\prod_{j=p+1}^{q} C_{j} \rightarrow I$ as $q>p \rightarrow \infty$, thus there is an $N_{2}$ such that $n \geq N_{2}$ implies

$$
\left|L_{2}^{-1}\right|\left|\prod_{j=n+1}^{\infty} C_{j}-I\right|\left|L_{1}\right|<\frac{\epsilon}{2} .
$$

Here, the Cauchy-Schwarz inequality gives

$$
\left|\left(\prod_{j=1}^{n} C_{j}\right)^{-1} L_{1}-L_{2}^{-1} L_{1}\right|<\frac{\epsilon}{2}
$$

For $n \geq N=\max \left\{N_{1}, N_{2}\right\}$, adding Eq.(4.16) to Eq.(4.17) and applying the triangle inequality establishes the first statement of the theorem. This fact, along with assumption (iii) and [2, Theorem 6.1] yields the second statement and completes the proof.

The application to continued fractions is an immediate corollary.

Corollary 4.4 If $\widehat{U}_{n} \rightarrow \widehat{U}_{\infty}$ and $\prod_{j=2}^{n} K_{j} \rightarrow \mathcal{K}_{\infty}$ where both $\widehat{U}_{\infty}$ and $\mathcal{K}_{\infty}$ are nonsingular, then $\mathcal{U}_{n} \rightarrow \mathcal{K}_{\infty}^{-1} \widehat{U}_{\infty}$ and $\mathcal{Y}_{n} \rightarrow \mathcal{Y}^{\infty}$, a finite matrix. 
Remark 4.5 (parameter qualifications) Before proceeding we summarize our strategy and the parameter restrictions. The leading order behavior of the matrix expansions in powers of $n^{-1}$ is guaranteed by the boundedness assumptions on the parameters $a_{n}$ with which we began this work. Subsequent restrictions were added in order to constrain the matrix products describing the dynamics of the related difference equation. The strongest restrictions on the sequences $\left(a_{n}\right)$ come from the invertibility assumption in Theorem 4.1 and that of $\widehat{U}_{\infty}$ and $\mathcal{K}_{\infty}$ in Theorem 4.4. The first of these, that $\operatorname{det}\left(\mathcal{Y}^{\infty}\right) \neq 0$ is discussed in [5, Remark 4.2] and is needed to assure the separation of the odd and even convergents of $\mathcal{S}_{1}$. The assumptions on the invertibility of $\prod_{j=1}^{n} K_{j} \rightarrow \mathcal{K}_{\infty}$ and $\widehat{U}_{\infty}$ were shown in [5, Remark 4.4] to be equivalent, respectively, to

$$
\begin{gathered}
0 \neq \lim _{n \rightarrow \infty} \frac{\alpha_{2}^{1 / 2}}{\alpha_{2 n}^{n-1 / 2} \alpha_{2 n-1}^{n-1}} \prod_{j=1}^{2 n-2} \alpha_{j}<\infty \\
0 \neq \prod_{j=2}^{\infty}\left(1-\frac{1}{\left(2 j a_{2 j}\right)^{2}}\right)<\infty .
\end{gathered}
$$

\subsection{Exponential-sums}

The problem of determining the convergence or divergence of $\mathcal{S}_{1}$ has been reduced to determining

the convergence or divergence of $\widehat{U}_{n}$ defined by Eq.(4.15). In [2] an exponential sum analysis was applied to such matrix products for the case of cycles of length $c=2$ in order to obtain detailed results about the convergence of $\widehat{U}_{n}$. Though this analysis does not appear to be tractable in general, we set out the formal basis from which useful special cases may be gleaned.

To begin, note that even products of $\widehat{W}_{j}$ are diagonal matrices, and odd products are skew matrices. To wit, we have

$$
\prod_{j=1}^{2 n+1} \widehat{W}_{j}=\left(\prod_{j=1}^{2 n+1} \frac{1}{a_{2 j}}\right)\left[\begin{array}{cc}
0 & \prod_{j=1}^{2 n+1} \omega_{j}^{\left(-1^{j+1}\right)} \\
\prod_{j=1}^{2 n+1}\left(\omega_{j}^{-1}\right)^{\left(-1^{j+1}\right)} & 0
\end{array}\right]
$$


while

$$
\prod_{j=1}^{2 n} \widehat{W}_{j}=\left(\prod_{j=1}^{2 n} \frac{1}{a_{2 j}}\right)\left[\begin{array}{cc}
\prod_{j=1}^{2 n} \omega_{j}^{\left(-1^{j}\right)} & 0 \\
0 & \prod_{j=1}^{2 n}\left(\omega_{j}^{-1}\right)^{\left(-1^{j}\right)}
\end{array}\right]
$$

Let $\underline{\omega}_{n}=\left(\omega_{1}, \ldots, \omega_{n}\right)$ and

$$
\mathcal{T}_{j}\left(n, \underline{\omega}_{n}\right):=\sum_{m_{j}>\cdots>m_{1} \geq 1}^{n}\left(\prod_{k=1}^{j} \frac{\omega_{m_{k}}^{\left(-1^{(j \bmod 2)+k}\right)}}{m_{k} a_{2 m_{k}}}\right)
$$

where the sum is empty (0) if $j>n$ and, by definition, $\mathcal{T}_{0}\left(n, \underline{\omega}_{n}\right):=1$. In general we have

$$
\widehat{U}_{n}=I+\sum_{j=1}^{n}\left(\sum_{m_{j}>\cdots>m_{1} \geq 1}^{n}\left(\prod_{k=1}^{j} \frac{1}{2 m_{k}} \widehat{W}_{m_{k}}\right)\right)=\left[\begin{array}{cc}
\mathcal{A}_{n}\left(\underline{\omega}_{n}\right) & \mathcal{B}_{n}\left(\underline{\omega}_{n}\right) \\
\mathcal{B}_{n}\left(\underline{\omega}_{n}^{-1}\right) & \mathcal{A}_{n}\left(\underline{\omega}_{n}^{-1}\right)
\end{array}\right],
$$

where

$$
\mathcal{A}_{n}\left(\underline{\omega}_{n}\right):=\sum_{j=0}^{\infty} 2^{-2 j} \mathcal{T}_{2 j}\left(n, \underline{\omega}_{n}\right) \quad \text { and } \quad \mathcal{B}_{n}\left(\underline{\omega}_{n}\right):=\sum_{j=0}^{\infty} 2^{-2 j} \mathcal{T}_{2 j+1}\left(n, \underline{\omega}_{n}\right) .
$$

This formulation is difficult to work with in general, though for some special cases it yields explicit bounds on matrix elements as the next example illustrates.

Example 4.6 Let $a_{n}=a_{n+2} \quad(n=1,2, \ldots)$. From Eq.(4.12) we have

$$
\omega_{n}=\frac{a_{2 n}}{a_{2}} \prod_{j=1}^{n} \frac{\alpha_{2 j-1}}{\alpha_{2 j}}=\left(\frac{\alpha_{1}}{\alpha_{2}}\right)^{n}
$$

which we will write as $\omega^{n}$. Thus

$$
\mathcal{T}_{j}(n, \underline{\omega})=\frac{1}{a_{2}^{j}} \sum_{m_{j}>\cdots>m_{1} \geq 1}^{n}\left(\prod_{k=1}^{j} \frac{\omega^{\left(-1^{(j \bmod 2)+k}\right) m_{k}}}{m_{k}}\right) .
$$


This form of the exponential sum, it turns out, is tractable. Indeed, this can be rewritten as

$$
\begin{aligned}
& \mathcal{T}_{j}(n, \underline{\omega})= \\
& \quad\left(\prod_{k=1}^{j} \omega^{\left(-1^{(j \bmod 2)+k}\right)}\right) \int_{0}^{1} \cdots \int_{0}^{1} d x_{1} \cdots d x_{j} S_{j}\left(n ; \omega^{\left(-1^{(j \bmod 2)+1}\right)} x_{1}, \ldots, \omega^{\left(-1^{(j \bmod 2)+j}\right)} x_{j}\right),
\end{aligned}
$$

where

$$
S_{j}\left(n ; z_{1}, \ldots, z_{j}\right)=\sum_{m_{j}>\cdots>m_{1} \geq 1}^{n} z_{n}^{m_{j}} z_{j-1}^{m_{j-1}} \ldots z_{1}^{m_{1}} .
$$

In particular,

$$
S_{j}\left(\infty ; z_{1}, \ldots, z_{j}\right)=\frac{z_{j}^{j-1}}{1-z_{j}} \frac{z_{j-1}^{j-2}}{1-z_{j} z_{j-1}} \cdots \frac{1}{1-z_{j} z_{j-1} \cdots z_{1}} .
$$

Using these identities, it can been shown that if $|\omega|=1$ with $\omega \neq 1$, then the matrix $\mathcal{U}_{n}=\widehat{U}_{n}$ converges as $N \rightarrow \infty$ with explicit bounds on the limit $\mathcal{U}_{\infty}$ (see [2, Theorem 7.5]).

An interesting open problem is to find an integral representation similar to Eq.(4.23) for generalized Lerch sums of the form Eq.(4.22) with parameters $\underline{\omega}=\left(\omega_{1}, \ldots, \omega_{n}\right)$ involving more complicated behavior. Given the simplicity of $w_{n}$ given by Eq.(4.12), it seems quite likely that tractable reformulations can be extracted from Eq.(4.22).

\subsection{General Matrix Analysis}

Given the difficulty of working with generalized exponential sums of the form Eq.(4.22), we pursue a more general approach. The following is a special, deterministic case of [5, Theorem 4.5]. 
Theorem 4.7 (matrix products) Let the sequences $\left(\zeta_{j}\right)$ and $\left(\zeta_{j}^{\prime}\right)$ satisfy

$$
\sup _{k}\left|\sum_{j \geq n}^{k} \zeta_{j}\right|<\infty \quad \text { and } \quad \sup _{k}\left|\sum_{j \geq n}^{k} \zeta_{j}^{\prime}\right|<\infty
$$

and let $\left(\eta_{j}\right)$ be a real nonnegative square summable sequence decreasing monotonically to 0 . Then the matrix product

$$
\prod_{j=1}^{n}\left(I+\eta_{j}\left[\begin{array}{cc}
0 & \zeta_{j} \\
\zeta_{j}^{\prime} & 0
\end{array}\right]\right)
$$

converges to a finite matrix as $n \rightarrow \infty$. If, in addition,

$$
\left|1-\eta_{j}^{2} \zeta_{j}^{\prime} \zeta_{j}\right| \geq m>0 \quad \forall j
$$

then the matrix product converges invertibly.

Remark 4.8 Compare this result to a similar result by Trench [7, Theorem 4] which states that for any sequence of $m \times m$ matrices $\left(A_{n}\right)$ the product $\prod_{n=1}^{\infty}\left(I+A_{n}\right)$ converges invertibly if $\sum^{\infty}\left|A_{n}\right|<$ $\infty$. Condition Eq.(4.24) is less restrictive than the requirement that the corresponding matrix norms be summable, however our result is not as general. In light of other sufficient conditions developed by Trench [7, Theorems 5-6] it would be interesting to see if the techniques presented here can be applied to more general matrix products.

Odd cycles/arbitrary sequences: proof of Theorem 1.1(i), $b=2$. Consider the case of odd-length cycles or, more generally, arbitrary sequences $\left(a_{n}\right)$. Here, for $c \in \mathbb{N} \cup\{\infty\}$ not even,

$$
\zeta_{n}=\zeta_{n \bmod c}:=\frac{1}{a_{2 n \bmod c}} \omega_{n}=\frac{1}{a_{2}}\left(\prod_{j=1}^{n \bmod c} \frac{\alpha_{2 j-1}}{\alpha_{2 j}}\right) .
$$


For the inverse, we have

$$
\zeta_{n}^{\prime}=\zeta_{n \bmod c}^{\prime}:=\frac{1}{a_{2 n \bmod c}} \omega_{n}^{-1}=\frac{a_{2}}{\alpha_{2 n \bmod c}}\left(\prod_{j=1}^{n \bmod c} \frac{\alpha_{2 j-1}}{\alpha_{2 j}}\right)^{-1}
$$

(see Eq.(4.14)). If $\zeta_{n}$ and $\zeta_{n}^{\prime}$ satisfy

$$
\sup _{k}\left|\sum_{n=1}^{k} \zeta_{n}\right|<\infty, \quad \text { and } \sup _{k}\left|\sum_{n=1}^{k} \zeta_{n}^{\prime}\right|<\infty
$$

then Theorems 4.1, 4.4, and 4.7 yield part (ii) of Theorem 1.1 for $b=2$.

Example 4.9 Condition Eq.(1.2), while nontrivial, is not difficult to satisfy. It is certainly satisfied by any sequence $\left(\zeta_{n}\right)$ which processes "evenly" around the unit circle in the complex plane, or, in the case of random sequences, any bounded $\left(\zeta_{n}\right)$ with mean equal to zero ${ }^{2}$. We have already seen instances of such sequences in Section 2. For example, for the case of $\left(a_{n}\right)$ with odd-length cycles, say $c=3$, then

$$
\zeta_{1}=\frac{a_{1}^{2}}{a_{2}^{3}}, \quad \zeta_{2}=\frac{a_{3}^{2}}{a_{2}^{3}} \quad \text { and } \quad \zeta_{3}=\frac{1}{a_{2}}
$$

while

$$
\zeta_{1}^{\prime}=\frac{a_{2}}{a_{1}^{2}}, \quad \zeta_{2}^{\prime}=\frac{a_{2}^{3}}{a_{1}^{2} a_{3}^{2}} \quad \text { and } \quad \zeta_{1}^{\prime}=\frac{a_{2}}{a_{3}^{2}}
$$

The parameters $a_{i}$ then must satisfy

$$
a_{1}^{2}+a_{2}^{2}+a_{3}^{2}=0
$$

In Fig. 5 the parameters for iteration Eq.(2.1) with a cycle length 3 are $\left(a_{1}, a_{2}, a_{3}\right)=$

\footnotetext{
${ }^{2}$ Note that the case of random $\zeta_{n}$ is more delicate since the partial sums Eq.(4.29) are not bounded above.
} 


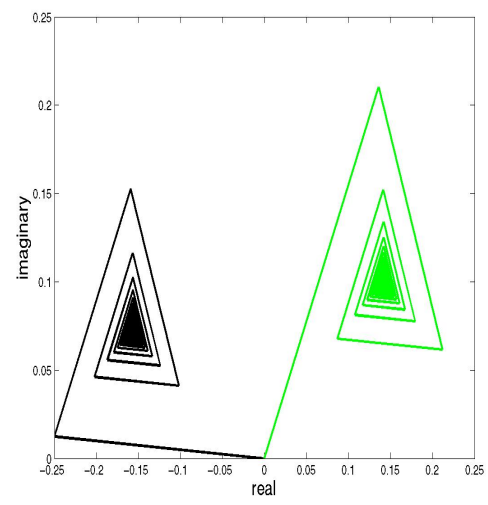

Figure 7: Sequence of partial sums given by Eq.(4.30) for a cycle length 3 with parameters $\left(a_{1}, a_{2}, a_{3}\right)=(\exp (\mathrm{i} \pi / 2), \exp (\mathrm{i} \pi / 6), \exp (-\mathrm{i} \pi / 6))$ corresponding to Fig. 5. The dark line corresponds to the partial sums of $\frac{1}{a_{2 j}} \omega_{j}$ and the light line to the partial sums of $\frac{1}{a_{2 j}} \omega_{j}^{-1}$.

$(\exp (\mathrm{i} \pi / 2), \exp (\mathrm{i} \pi / 6), \exp (-\mathrm{i} \pi / 6))$ The dynamics of the corresponding sequence of partial sums,

$$
\left(\sum_{j=1}^{n} \frac{1}{2 j a_{2 j}} \omega_{j}\right) \quad \text { and } \quad\left(\sum_{j=1}^{n} \frac{1}{2 j a_{2 j}} \omega_{j}^{-1}\right)
$$

is depicted in Fig. 7. In Fig. 6 the parameters are $a_{1}=\pi / 3+0.05, a_{2}=-\pi / 3+0.05$, and $a_{3}=0.05$. The dynamics of the corresponding sequence of partial sums are similar to those shown in Fig. 7.

Even cycles: proof of Theorem 1.1(ii), $b=2$. Let $a_{j}=a_{j+c}$ for all $j$ and $c$ finite and even. Define $\omega_{n}$ by Eq.(4.13), and define

$$
\zeta_{k}:=\frac{1}{a_{2 k}} \omega_{k}=\gamma^{\lfloor 2 k / c\rfloor} \xi_{k} \quad \text { where } \quad \xi_{k}=\xi_{k \bmod c / 2}:=\frac{1}{a_{2}}\left(\prod_{j=1}^{k \bmod c / 2} \frac{\alpha_{2 j-1}}{\alpha_{2 j}}\right)
$$

and

$$
\gamma:=\left(\prod_{j=1}^{c / 2} \frac{\alpha_{2 j-1}}{\alpha_{2 j}}\right)
$$


Similarly, define

$$
\zeta_{k}^{\prime}:=\frac{1}{a_{2 k}} \omega_{k}^{-1}=\gamma^{-\lfloor 2 k / c\rfloor} \xi_{k}^{\prime} \quad \text { where } \quad \xi_{k}^{\prime}=\xi_{k \bmod c / 2}^{\prime}:=\frac{a_{2}}{\alpha_{2 k \bmod c}}\left(\prod_{j=1}^{k \bmod c / 2} \frac{\alpha_{2 j-1}}{\alpha_{2 j}}\right)^{-1}
$$

The next Lemma yields the specialization (ii) of Theorem 1.1 for $b=2$.

Lemma 4.10 Let $\xi_{j}, \xi_{j}^{\prime} \in \mathbb{C}$ satisfy $\left|\xi_{j}\right| \leq z<\infty,\left|\xi_{j}^{\prime}\right| \leq z^{\prime}<\infty \forall j$ and let $|\gamma|=1$ with $\gamma \neq 1$. Then for any positive $d \in \mathbb{N}$, we have

$$
\sup _{k}\left|\sum_{j=0}^{k} \gamma^{\lfloor j / d\rfloor} \xi_{j}\right|<\infty \quad \text { and } \quad \sup _{k}\left|\sum_{j=0}^{k} \gamma^{-\lfloor j / d\rfloor} \xi_{j}^{\prime}\right|<\infty
$$

Proof. This follows immediately for $d$ finite and $\gamma \neq 1$ since $\left|\xi_{j}\right| \leq z<\infty$ and $\left|\xi_{j}^{\prime}\right| \leq z^{\prime}<\infty$ for all $j$ and $\gamma^{ \pm j}$ is a (nonstationary) rotation around the unit disk in $\mathbb{C}$.

If $|\gamma|=1$ with $\gamma \neq 1$ and if $\left(a_{n}\right)$ satisfies Eq.(4.18)-(4.19) then Theorems 4.1, 4.4, and 4.7, together with Lemma 4.10 complete the proof of Theorem 1.1(ii) with $b=2$.

Example 4.11 The dynamics of the sequence of partial sums corresponding to iteration Eq.(2.1) for a cycle length 4 with parameters $\left(a_{1}, a_{2}, a_{3}, a_{4}\right)=(\exp (\mathrm{i} \pi / 4), \exp (\mathrm{i} \pi / 6), \exp (\mathrm{i} \pi / 4), \exp (\mathrm{i}(\pi / 6+$ 1/2))) (see Fig. 2) is depicted in Fig. 8.

\section{Concluding Remarks and Open Problems}

While the principal application of interest in this work has been the determination of the divergence of continued fractions, our analysis touches on many different areas of mathematics, from difference 


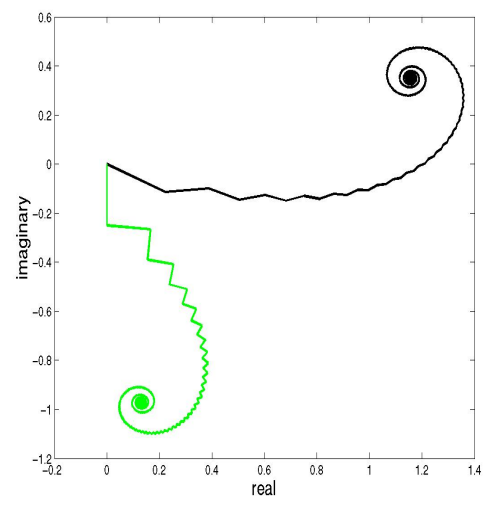

Figure 8: Sequence of partial sums given by Eq.(4.30) for a cycle length 4 with parameters $\left(a_{1}, a_{2}, a_{3}, a_{4}\right)=(\exp (\mathrm{i} \pi / 4), \exp (\mathrm{i} \pi / 6), \exp (\mathrm{i} \pi / 4), \exp (\mathrm{i}(\pi / 6+1 / 2)))$ corresponding to Fig. 2. The light line corresponds to the partial sums of $\frac{1}{a_{2 j}} \omega_{j}$ and the dark line to the partial sums of $\frac{1}{a_{2 j}} \omega_{j}^{-1}$.

equations, to dynamical systems, to matrix theory. We noted in Section 4.2 a direction for further research is to find an integral representation similar to Eq.(4.23) for generalized Lerch sums of the form Eq.(4.22) with parameters $\underline{\omega}=\left(\omega_{1}, \ldots, \omega_{n}\right)$ involving more complicated behavior. While the continued fractions we considered here lead only to 2 term difference equations such as Eq.(1.3), one could conceive of more general difference equations in and of themselves, for example recursions of the form

$$
q_{n}=(n+1-m) \alpha_{n}\left(\begin{array}{c}
n \\
n-m
\end{array}\right) q_{n-m-1}+\sum_{j=0}^{m-1}\left(\begin{array}{c}
n \\
n-j
\end{array}\right) q_{n-(j+1)}
$$

and their corresponding renormalized difference equations

$$
t_{n+1}=\frac{n+1-m}{n+1} \alpha_{n} t_{n-m}+\frac{1}{n+1} \sum_{j=0}^{m-1} t_{n-(j+1)}
$$

Such generalizations would lead to an $(m+1) \times(m+1)$ matrix analysis analogous to that pursued here. One possibly far reaching issue is whether or not the general recurrence relations above admit generating functions. If so, what can be said about these generating functions, the sequences they encode and the functions they characterize? 
Finally, the attentive reader will note that we have left out any mention of the parameter values corresponding to the simulations shown in Fig. 3 and Fig. 4. It is easy to verify that for the parameter values in these examples the partial sums corresponding to Eq.(4.29) are not bounded. However, condition Eq.(4.29) is only sufficient, thus we cannot determine from our analysis whether or not the continued fraction $\mathcal{S}_{1}$ converges for these parameter values. Our analysis, while quite general, still leaves undetermined the necessary conditions for the matrix products $\mathcal{Y}_{n}$ to converge.

\section{Acknowledgments}

We thank an anonymous referee, Ian Coope and Richard Crandall for many valuable comments. Russell Luke's work was supported in part by a postdoctoral fellowship from the Pacific Institute for the Mathematical Sciences at Simon Fraser University.

\section{References}

[1] M. Abramowitz and I. A. Stegun, editors. Handbook of Mathematical Functions. Dover, New York, 9th edition, 1972.

[2] D. Borwein, J. M. Borwein, R. Crandall, and R. Mayer. On the dynamics of certain recurrence relations. Ramanujajn Journal, 2004. in press.

[3] J. M. Borwein and R. Crandall. On the Ramanujan AGM fraction. part II: The complex parameter case. Experimental Mathematics, 13:287-296, 2004.

[4] J. M. Borwein, R. Crandall, and G. Fee. On the Ramanujan AGM fraction. part I: The real parameter case. Experimental Mathematics, 13:275-286, 2004.

[5] J. M. Borwein and D. R. Luke. Dynamics of a continued fraction of Ramanujan with random coefficients. Abstract and Applied Analysis, 5:449-467, 2005. 
[6] L. Lorentzen and H. Waadeland. Continued Fractions with Applications. North-Holland, New York, 1992.

[7] W. F. Trench. Invertibly convergent infinite products of matrices. J. Comp. Appl. Math., pages 255-63, 1999. 


\section{Footnotes}

* Jonathan M. Borwein, Faculty of Computer Science, Dalhousie University, Halifax, Nova Scotia B3H 1W5, Canada E-mail: jborwein@cs.dal.ca

$\dagger$ D. Russell Luke Department of Mathematical Sciences, University of Delaware, Newark, DE 19716-2553 E-mail: rluke@math.udel.edu

1. We define empty products to be equal to 1.

2. The reason we cannot immediately extend these results to random sequences is because the partial sums Eq.(4.29) are not bounded above for such sequences. 